\title{
Application of the chronic constriction injury of the partial sciatic nerve model to assess acupuncture analgesia
}

This article was published in the following Dove Press journal:

Journal of Pain Research

19 September 2017

Number of times this article has been viewed

\author{
Mu-Jun Zhi' ${ }^{1,2, *}$ \\ Kun Liu',* \\ Zhou-Li Zheng',3 \\ Xun $\mathrm{He}^{\prime}$ \\ Tie $\mathrm{Li}^{2}$ \\ Guang Sun ${ }^{1,2}$ \\ Meng Zhang ${ }^{4}$ \\ Fu-Chun Wang ${ }^{2}$ \\ Xin-Yan Gao' \\ Bing Zhu'
}

'Department of Physiology, Institute of Acupuncture and Moxibustion, China Academy of Chinese Medical Sciences, Beijing, People's Republic of China; ${ }^{2}$ College of Acupuncture and Moxibustion, Changchun University of Chinese Medicine, Changchun, People's Republic of China; ${ }^{3}$ College of Acupuncture and Moxibution, Shaanxi University of Chinese Medicine, People's Republic of China; ${ }^{4}$ Department of Chinese Medicine, Dongli Hospital of Traditional Chinese Medicine, Tianjin, People's Republic of China

*These authors contributed equally to this work

Correspondence: Bing Zhu; Xin-Yan Gao Department of Physiology, Institute of Acupuncture and Moxibustion, China Academy of Chinese Medical Sciences, I6 Nanxiaojie, Dongzhimennei, Beijing 100700, People's Republic of China Tel +86 I0 6408 9275; +861064089420 Email zhubing@mail.cintcm.ac.cn; gaoxy@mail.cintcm.ac.cn
Purpose: To validate and explore the application of a rat model of chronic constriction injury to the partial sciatic nerve in investigation of acupuncture analgesia.

Methods: Chronic constriction injury of the sciatic nerve (CCI) and chronic constriction injury of the partial sciatic nerve (CCIp) models were generated by ligating either the sciatic nerve trunk or its branches in rats. Both models were evaluated via paw mechanical withdrawal latency (PMWL), paw mechanical withdrawal threshold (PMWT), nociceptive reflex-induced electromyogram (C-fiber reflex EMG), and dorsal root ganglion immunohistochemistry. Electroacupuncture (EA) was performed at GB30 to study the analgesic effects on neuropathic pain and the underlying mechanisms.

Results: Following ligation of the common peroneal and tibial nerves, CCIp rats exhibited hindlimb dysfunction, hind paw shrinkage and lameness, mirroring those of CCI rats (generated by ligating the sciatic nerve trunk). Compared to presurgery measurements, CCIp and CCI modeling significantly decreased the PMWL and PMWT. EA at GB30 increased the PMWL and PMWT in both CCI and CCIp rats. Calcitonin gene-related polypeptide and substance P expressions were apparently increased in both CCI and CCIp groups, but were not different from each other. The C-fiber reflex EMG of the biceps femoris was preserved in CCIp rats, but it could not be recorded in CCI rats on the 5th day after nerve ligation. The C-fiber reflex EMG was reduced at 0,1 , and 2 minutes after EA in CCIp rats, but only at 0 and 1 minute after EA in normal rats.

Conclusion: The CCIp model is better than the CCI model for studying acupuncture analgesia on chronic neuropathic pain and the underlying mechanisms.

Keywords: neuropathic pain, chronic constriction injury, acupuncture, analgesia, C-fiber reflex

\section{Introduction}

Neuropathic pain, defined by the International Association for the Study of Pain as "pain caused by a lesion or disease of the somatosensory system", 1 is a pain syndrome characterized by spontaneous pain, hyperalgesia, allodynia, and sensory abnormalities. ${ }^{2}$ Sciatica, one of the most common sources of clinical neuropathic pain, is generally defined as pain radiating to the leg, normally below the knee and into the foot and toes. ${ }^{3}$ The treatment for sciatica remains a current challenge in clinic. Therefore, generating appropriate animal models are critical to developing novel treatment options for neuropathic pain. ${ }^{46}$

Chronic constriction injury of the sciatic nerve (CCI) was established in 1988 as a means to produce neuropathic pain in laboratory animal models $;^{7}$ the pathological condition of this model is quite similar to human peripheral nerve injury and its consequent 
behavioral manifestations. Based on the CCI model, a variety of animal models have since been developed for different research purposes, including the partial sciatic nerve ligation (pSNL), ${ }^{8}$ the spinal nerve ligation to sciatic nerve (SNL), ${ }^{9}$ the sciatic nerve axotomy, ${ }^{10}$ the chronic compression of the dorsal root ganglion, ${ }^{11,12}$ and the spared nerve injury (SNI) models. ${ }^{13}$

Although basic and clinical studies have confirmed that acupuncture can effectively alleviate or cure pain syndromes, ${ }^{14,15}$ the mechanisms responsible for acupuncture analgesia remain elusive. This suggests the need for a proper animal model in order to elucidate mechanisms by which acupuncture alleviates neuropathic pain. Behavior test and nociceptive stimulation-induced EMG (C-fiber reflex EMG) are both measures for pain alleviating evaluation. Paw mechanical withdrawal latency and threshold (PMWL and PMWT, respectively) are the tests of mechanical nociception or sensibility based on the rat's sensory-motor reflex. It is withdrawal of the paw with participation of the upper central nervous system and multisynaptic relay. Whereas $\mathrm{C}$-fiber reflex is a neuromuscular reflex with the participation of the spinal level and usually is a three-synapse reflex, which is a more objective and direct criterion in evaluation of pain and acupuncture analgesia. ${ }^{16-18}$ Given that acupuncture may inhibit the C-fiber reflex EMG, the mechanisms underlying acupuncture analgesia may be explained at both the peripheral nervous system level as well as the spinal central nervous system level.

Different sciatic nerve compression or ligation models could induce different influences on $\mathrm{C}$-fiber reflexes. The CCI model can cause a diffused lesion on efferent fibers and thus damage muscle innervation of the sciatic nerve. Because damage to the motor nerve and muscular atrophy in the area innervated by the sciatic nerve may result in failure of pain behaviors and C-fiber reflex recordings (thereby affecting the evaluation of acupuncture analgesia), we aimed to establish a novel animal model to evaluate $\mathrm{C}$-fiber reflex changes induced by acupuncture: namely, chronic constriction injury of the partial sciatic nerve (CCIp).

In this study, we compared the characteristics of CCIp and CCI models and utilized the CCIp model to evaluate acupuncture analgesia. In addition, we investigated the correlation between analgesia of acupoint and the location of sciatic nerve compression.

\section{Materials and methods}

\section{Animals}

This study was carried out in strict accordance with the recommendations in the Guide for the Care and Use of
Laboratory Animals of the National Institutes of Health. The protocol was approved by the Committee on the Ethics of Animal Experiments of China Academy of Chinese Medical Sciences (Approval Number: AE20110510-001). Male adult Sprague Dawley rats (200-250 g) were purchased from the Military Academy of Medical Sciences [Animal Certificate of Conformity: SCXK (Army) 2012-0004] (Beijing, People's Republic of China). Rats were housed in clean animal facilities with the room temperature maintained at $24^{\circ} \mathrm{C} \pm 0.5^{\circ} \mathrm{C}$, humidity at $60 \%-70 \%$, and noise levels lower than $60 \mathrm{~dB}$. All animals were housed in groups of two or three with ad libitum access to food and water. The animals were maintained on a standard 12-hour light-dark cycle (dark cycle 8:00 PM-8:00 AM) and were allowed to acclimate to the housing conditions for 7 days prior to the experiment.

\section{Surgical preparation}

Animals were anesthetized with $10 \%$ urethane $(1.0-1.2 \mathrm{~g} / \mathrm{kg}$, intraperitoneal) and placed on a sterile operating table in the left-lateral position. Before surgery, the 4-0 chromic gut (MY 41839, MYCO Medical Supplies Inc., Cary, NC, USA) was soaked in normal saline for at least 30 minutes. $^{7}$ After shaving and sterilizing the right leg with iodophor, a transverse incision was made underneath the long head of biceps femoris and the sciatic nerve was exposed at the level of the middle of the thigh by blunt dissection for CCI of sciatic nerve trunk. And for CCIp, an oblique incision of the thigh through the skin and muscle was made and the sciatic nerve and its three terminal branches (sural, common peroneal, and tibial nerves) were exposed. Also by blunt dissection, these branches were isolated.

Four ligatures were made in both the CCI and the CCIp models. Proximal to the sciatic nerve's trifurcation, $\sim 7 \mathrm{~mm}$ of nerve was freed from the adhering tissue, and ligatures were tied around it with $\sim 1 \mathrm{~mm}$ spacing in the CCI model (Figure 1A). ${ }^{7}$ Four ligatures were tied around the tibial nerve and the common peroneal nerve in CCIp model (Figure 1B) modified from the pSNL and SNI model. ${ }^{8,13}$ Great care was taken to avoid any damage to the sural nerve. Nerve compression was made tight enough so that toes on the fibula side twitched slightly. The innervation of the gastrocnemius was influenced by compression injury. Muscle and skin were sutured separately and carefully in layers. The neurotrophy and cutaneous sensation of the tissue were maintained as well as possible. The phenotypes of spontaneous pain and mechanical hyperalgesia after nerve ligation included hindlimb discoordination, toes curling up, and limb lameness. 


\section{Pain measurement}

Mechanical pain thresholds were separately detected on the day before modeling and on the 5th and 17th day after modeling. Before pain assessment, rats were placed on the other side of a transparent partition with a stainless steel mesh bottom and allowed to move freely for 30 minutes. As modified from a previous report, ${ }^{19} \mathrm{PMWL}$ and PMWT were measured through a Dynamic Plantar Aesthesiometer (37450, UGO, Italy) with an ascending tip which exerted a force that increased at a constant rate of $2 \mathrm{~g} / \mathrm{s}$ with a cut-off force of $50 \mathrm{~g}$. Stimulus was delivered to the middle of the plantar hind paw between the second and third or third and fourth metatarsals via the tip (Figure 2). ${ }^{20}$ Each hind paw was tested three times with a 15 minutes interval between each measurement, and the three measured data were averaged.

\section{Immunohistochemistry}

Animals were anesthetized with $10 \%$ urethane $(1.0-1.2 \mathrm{~g} / \mathrm{kg}$, intraperitoneal) and transcardially perfused with $300 \mathrm{~mL}$
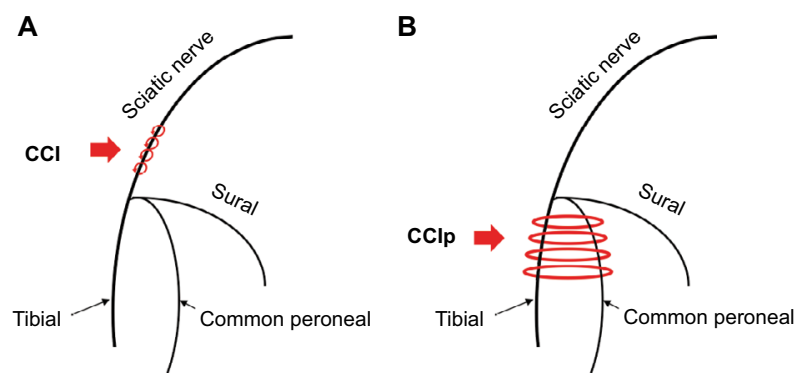

Figure I Schematic diagram of surgical ligation site in $\mathrm{CCl}$ and $\mathrm{CClp}$ rats.

Notes: (A) Four ligatures were tied around the sciatic nerve trunk with $\sim \mathrm{mm}$ space between ligatures in $\mathrm{CCl}$ rats. (B) Four ligatures were tied loosely around the tibial nerve and the common peroneal nerve in CClp rats.

Abbreviations: $\mathrm{CCl}$, chronic constriction injury of the sciatic nerve; $\mathrm{CClp}$, chronic constriction injury of the partial sciatic nerve.

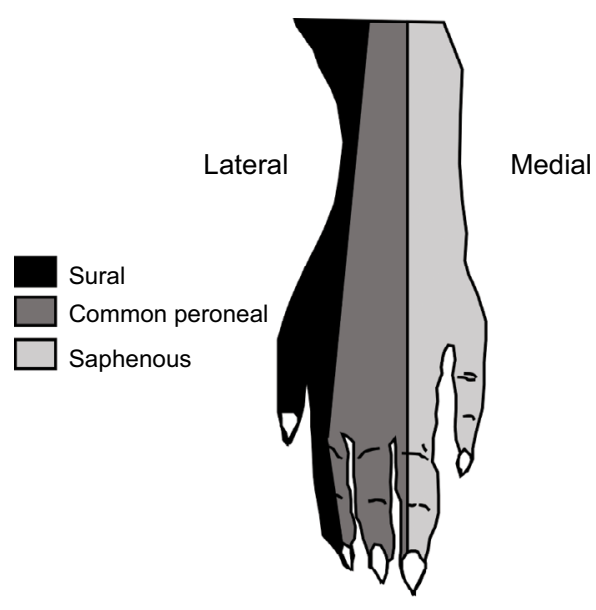

4\% paraformaldehyde in $0.1 \mathrm{M}$ phosphate-buffered saline (PBS), $\mathrm{pH}$ 7.4. The L4-L6 dorsal root ganglions (DRG) were removed and placed in the same fixative $\left(4^{\circ} \mathrm{C}\right)$ for 2 hours and then kept in $0.1 \mathrm{M}$ PBS containing $25 \%$ sucrose for 48 hours. The DRG were cut into $40 \mu \mathrm{m}$ sections on a freezing microtome. Sections were placed in $0.01 \mathrm{M}$ PBS, washed three times, incubated in $0.3 \%$ Triton X-100 for 30 minutes, rinsed 3 times in $0.01 \mathrm{M}$ PBS, then blocked in $10 \%$ goat serum for 1 hour. The tissue sections were incubated with a cocktail solution of primary antibodies containing calcitoningene-related peptide as a marker of neuro-peptidergic subpopulation (CGRP, mouse monoclonal, diluted 1:1000, ab81887, Abcam Trading (Shanghai) Company Ltd, Shanghai, People's Republic of China), substance $\mathrm{P}$ as a marker of neuro-peptidergic subpopulation (SP, rabbit polyclonal, diluted 1:500, ab67006, Abcam Trading (Shanghai) Company Ltd), and Isolectin B4 as a marker of non-peptidergic sensory fibers (goat polyclonal, diluted 1:1000, sc-31339, Santa Cruz Biotechnology Inc., Dallas, TX, USA) for 15 hours at $4^{\circ} \mathrm{C}$. Sections were washed three times with $0.01 \mathrm{M}$ PBS and then incubated in a cocktail solution of fluorescent secondary antibodies: donkey anti-rabbit IgG-conjugated Alexa Fluor 647 (diluted 1:500, ab150075, Abcam Trading (Shanghai) Company Ltd, red fluorescence), donkey anti-mouse IgGconjugated Alexa Fluor 488 (diluted 1:500, A21202, Invitrogen, Carlsbad, CA, USA, green fluorescence), and donkey anti-goat IgG-conjugated Alexa Fluor 405 (diluted 1:500, ab175664, Abcam Trading (Shanghai) Company Ltd, blue fluorescence) on a rocking platform shaker (protected from light) for 1 hour at room temperature. After washing three times with $0.01 \mathrm{M}$ PBS, the tissue sections were mounted on glass slides, dried at room temperature protected from

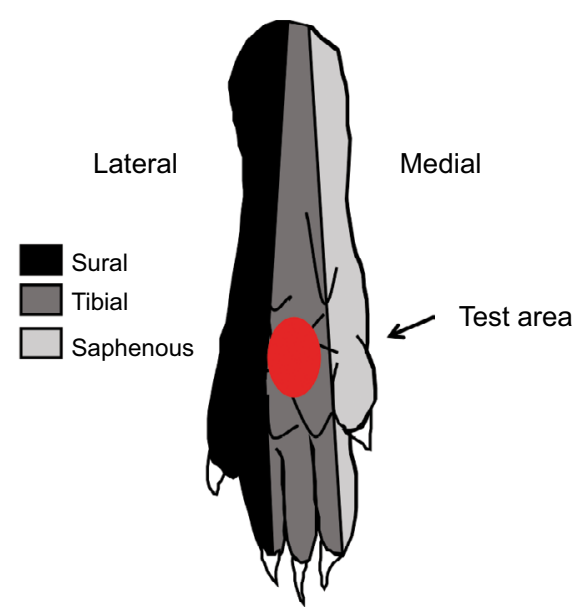

Figure $\mathbf{2}$ Neural receptive field and the area in rat plantar for the behavior test.

Notes: Left: neural receptive field in dorsum pedis of the hind paw. Right: neural receptive field in planta pedis of the hind paw. Red spot: the test area for mechanical pain threshold. 
light, and sealed with coverslips. Images of the DRG slices were acquired using a confocal laser scanning microscope (LMD6000, Leica, Wetzlar, Germany) with objective magnifications of $20 \times$ and $40 \times$. Laser channels used for excitation were $405 \mathrm{~nm}, 488 \mathrm{~nm}$, and $647 \mathrm{~nm}$ excitation, respectively.

\section{Electromyogram recording}

Electrophysiological recordings were performed before modeling and on the 5 th day after modeling. EMG of biceps femoris was recorded via a pair of noninsulated platinum-iridium electrodes. Electrical stimuli with single-square waves of $2 \mathrm{~ms}$ duration from Constant Current Isolated Stimulator (DS3, Digitimer Ltd., Welwyn Garden City, England) were delivered at a frequency of $0.2 \mathrm{~Hz}$ through stimulating electrodes which were placed in the third and fourth toes within the field of sural nerve innervation(Figure 2). ${ }^{13}$ Each stimulus was repeated 6 times. If no reflex was evoked by initial $0.5 \mathrm{~mA}$ intensity stimulation, a stepped stimulation with $0.1 \mathrm{~mA}$ was increased until the EMG was induced. The intervals between a set of six stimulation were 1 minute. EMG signals were amplified by Microelectrode Amplifier (SYS-DAM80, World Precision Instruments, Sarasota, FL, USA) and recorded by an acquisition system with Spike 2 (CED1401, Cambridge Electronic Design, Cambridge, $\mathrm{UK})$. The A $\delta$ fiber and $\mathrm{C}$-fiber reflex thresholds (Ta, Tc) were defined as the stimulation intensities required to evoke EMG of $\mathrm{A} \delta$ or C-fiber activities with the conductive velocity of 4-36 $\mathrm{m} / \mathrm{s}$ or $0.4-2 \mathrm{~m} / \mathrm{s}$, calculated by distances between the stimulus and recording sites dividing stable latency, respectively.

After Ta and Tc were recorded, EMG reflex induced by a 1.2 Tc stimulation was determined as the baseline for acupuncture analgesia evaluation. The C-fiber reflex EMG was recorded at $0,1,2,3$, and 4 minutes after 1-min electroacupuncture (EA) at GB30 (Huantiao) (intensities of $0.8 \mathrm{Ta}, 1$ Ta, 1 Tc or 2 Tc, randomly. The location and frequency of EA are described in detail in the "Electropuncture" section).

\section{Electroacupuncture}

The acupoint GB30 is located at the upper edge of hind hip, above the gluteus maximus muscle and stock side muscle beyond the compression site. ${ }^{21-23}$ After the animals were anesthetized with isoflurane gas, a disposable needle $(0.28$ $\mathrm{mm} \times 40 \mathrm{~mm}$ ) (Huatuo, Suzhou Medical Co. Ltd., Suzhou, People's Republic of China) was vertically inserted into GB30 to a depth of $7 \mathrm{~mm}$ and the reference electrode was placed $0.5 \mathrm{~cm}$ right lateral to the GB30. An electrical stimulus (15 Hz, $3 \mathrm{~mA}$ ) generated by HANS-200A analgesia apparatus (Nanjing Gensun Medical Technology Co. Ltd., Nanjing,
People's Republic of China) was delivered to GB30 for 10 minutes. During EA, the animals remained under isoflurane anesthesia. EA was conducted 6 times, once every 2 days for 2 weeks.

\section{Data analysis}

Data were displayed as mean \pm standard error of the mean and were analyzed using SPSS 16.0 (Statistical Package of Social Science, Chicago, IL, USA). C-fiber reflex recordings post-EA were normalized to that of the baseline pre-EA. Data with normal distributions were analyzed using the paired or unpaired $t$-test for two groups, and one-way or twoway analysis of variance for two or three groups. KruskalWallis test was used if the data did not satisfy homogeneity of variance. $P<0.05$ was considered to represent statistical significance.

\section{Results}

\section{Characteristics of $\mathrm{CCl}$ and CClp rats}

Animals in both the CCI and CCIp groups displayed spontaneous pain on the hind limb that underwent surgery. Symptoms of spontaneous pain were defined as hindlimb incoordination, toes curling up, and limb lameness. Three rats in CCI model group bit their own feet on the surgery side and were excluded from the study.

\section{Pain threshold in $\mathrm{CCl}$ and $\mathrm{CClp}$ rats}

As shown in Figure 3A, no difference in PMWL was found between CCI $(n=7)$ and CCIp $(n=10)$ rats before modeling. After ligating the sciatic nerve in CCI or two branches of the sciatic nerve in CCIp, the PMWL decreased significantly in both groups compared to their respective pre-model measurements (CCI: $16.64 \pm 0.84$ s for preligation vs $7.02 \pm 0.60$ s for postligation, $P<0.001$; CCIp: $16.86 \pm 0.57 \mathrm{~s}$ for preligation vs $7.75 \pm 0.55 \mathrm{~s}$ for postligation, $P<0.001)$. However, there was no significant difference between changes in both groups. As shown in Figure 3B, PMWT in CCIp rats was not significantly different from that in CCI rats before modeling (CCIp: $33.5 \pm 1.25 \mathrm{~g}$; CCI: $33.08 \pm 1.72 \mathrm{~g}$ ). PMWT in CCIp or CCI rats significantly decreased after nerve ligation (CCIp: $15.70 \pm 1.12 \mathrm{~g}$; CCI: $14.35 \pm 1.20 \mathrm{~g}$ ); however, no significant difference in PMWT was found between CCIp and CCI rats after modeling. These data suggest that ligation of either the trunk or the two branches of the sciatic nerve is capable of inducing hyperalgesia and producing neuropathic pain in the affected hind limb in rats; the phenotypes of CCIp are similar to those of CCI. 

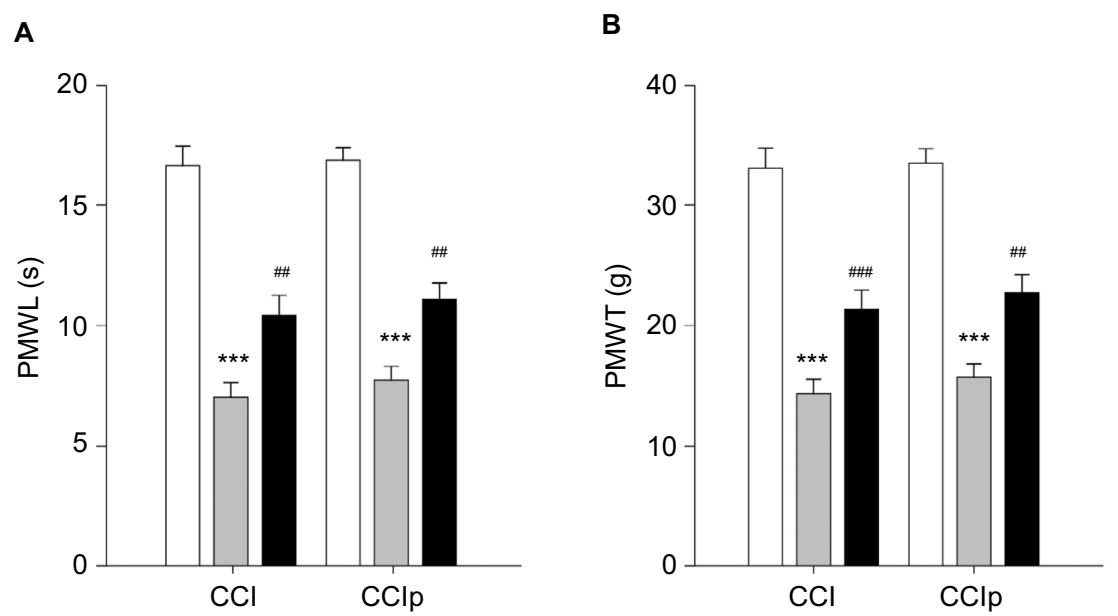

Figure 3 Effect of EA at GB30 on PMWL and PMWT in $\mathrm{CCl}$ and $\mathrm{CClp}$ rats.

Notes: (A) In either $\mathrm{CCl}(\mathrm{n}=7)$ or $\mathrm{CClp}(\mathrm{n}=10)$ rats, PMWL was decreased significantly on day 5 after nerve ligation (post-model) compared to I day before nerve ligation (pre-model) $\left({ }^{\infty} \mathrm{P}<0.00 \mathrm{I}\right.$, paired $t$-test). No significant difference was found between $\mathrm{CCl}$ and $\mathrm{CClp}$ rats (unpaired $t$-test). EA at $\mathrm{GB} 30$ caused significant increase in PMWL compared to post-model (\#P<0.0I, paired $t$-test); however, no significant difference was found between groups after EA at GB30 (unpaired $t$-test). (B) PMWT was significantly reduced after nerve ligation compared to one day before nerve ligation in $\mathrm{CCl}$ and $\mathrm{CCl} p$ rats ( ${ }^{* * * *} P<0.00 \mathrm{I}$, paired $t$-test). There was no significant difference between $\mathrm{CCl}$ and $\mathrm{CCl} p$ rats (unpaired $t$-test). EA at GB30 significantly augmented PMWT in both $\mathrm{CCl}$ (\#\#P<0.00I, unpaired $t$-test) and CClp rats $(\# P<0.0 \mathrm{I}$, paired $t$-test) compared to post-model, and there was no significant difference between both groups after EA at GB30 (unpaired t-test).

Abbreviations: $\mathrm{CCl}$, chronic constriction injury of the sciatic nerve; CClp, chronic constriction injury of the partial sciatic nerve; EA, electroacupuncture; PMWL, paw mechanical withdrawal latency; PMWT, paw mechanical withdrawal threshold.

A

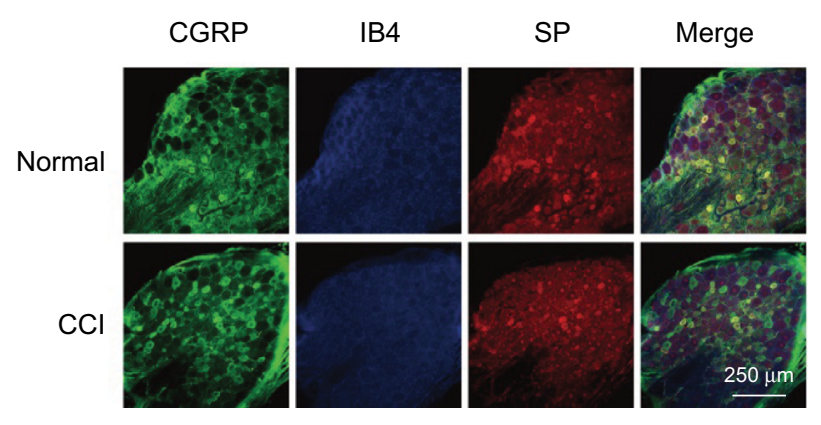

B

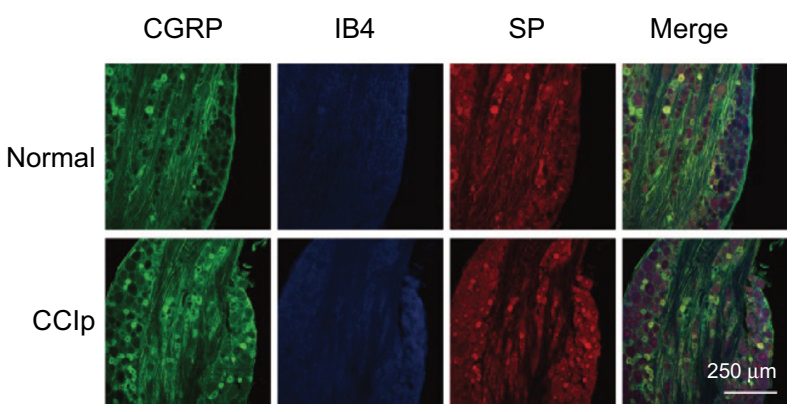

Figure 4 Expression of CGRP and SP in DRG neurons in $\mathrm{CCl}$ and $\mathrm{CClp}$ rats.

Notes: (A) Expression of CGRP and SP in DRG neurons in CCl rats, comparison of ipsilateral (CCI) to contralateral (normal). (B) Expression of CGRP and SP in DRG neurons in CClp rats, comparison of ipsilateral (CClp) to contralateral (normal). Scale bar: $250 \mu \mathrm{m}$.

Abbreviations: $\mathrm{CCl}$, chronic constriction injury of the sciatic nerve; CClp, chronic constriction injury of the partial sciatic nerve; CGRP, calcitonin-gene-related peptide; IB4, Isolectin B4; SP, substance P.

\section{Expression of CGRP and SP in DRG}

Subsequently, we observed expression of CGRP and SP in DRG of L4-6 ipsilateral compared to contralateral both in CCI and CCIp models. As shown in Figure 4A and B, CGRP and SP expression in normal rats were not apparently different between CCI and CCIp groups. After modeling, CGRP and SP expression were increased in CCI and CCIp rats. No difference was found between changes of CCI and CCIp groups (Figure 4A and B). These data suggest that ligation of the two branches of the sciatic nerve could increase the expression of CGRP and SP in a similar manner to the ligation of the trunk of the sciatic nerve.

\section{Fiber reflex}

We recorded EMG of the biceps femoris induced by Ta or Tc on the 5th day after nerve ligation. As shown in Figure 5A, EMG of the biceps femoris could be reliably induced by $\mathrm{Ta}$ or Tc in rats in the CCI and CCIp groups before nerve ligation. After ligating the sciatic nerve, EMG could not be triggered by Ta or Tc in CCI rats; however, in CCIp rats with sciatic branch as ligation, Tc could still induce a reliable biceps femoris EMG response while Ta failed to trigger an EMG response. As shown in Figure 5B, in CCIp $(n=10)$ rats, C-fiber reflex threshold decreased significantly as a result of nerve branch ligation (CCIp: $0.86 \pm 0.05 \mathrm{~mA}$, 
A

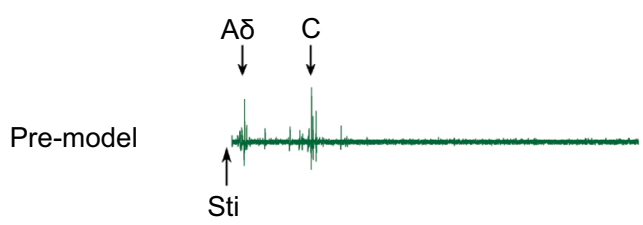

Post-model

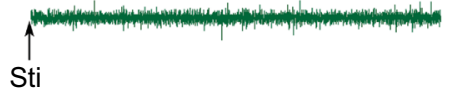

CClp

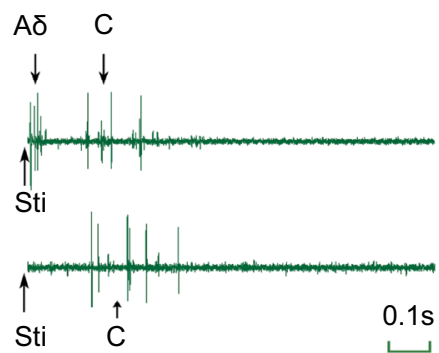

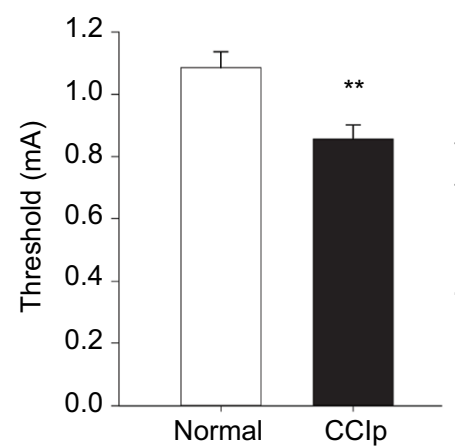

C

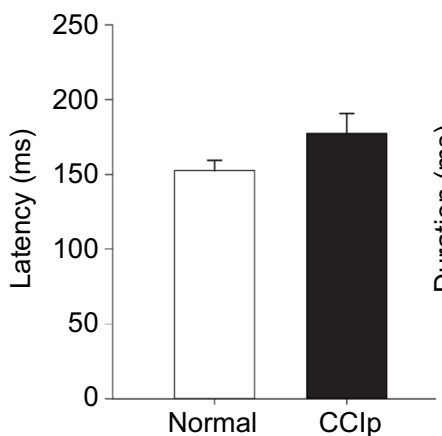

D

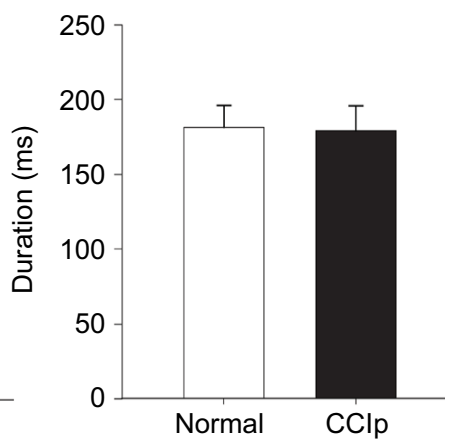

Figure 5 Threshold, latency, and duration of $\mathrm{A} \delta$ and $\mathrm{C}$-fiber reflex in $\mathrm{CCl}$ and $\mathrm{CClp}$ rats.

Notes: (A) Representative traces of EMG induced by $\mathrm{A} \delta$ fiber or $\mathrm{C}$-fiber reflex in $\mathrm{CCl}$ and $\mathrm{CClp}$ rats. Before nerve ligation (pre-model), both the A $\delta$ fiber and C-fiber stimulation induced independent EMG responses with different latencies in $\mathrm{CCl}$ and $\mathrm{CClp}$ rats. Five days after nerve ligation (post-model), neither the $\mathrm{A} \delta$ nor $\mathrm{C}$-fiber stimulation could induce EMG responses in $\mathrm{CCl}$ rats, whereas in CClp rats, the C-fiber reflex EMG could be induced, but the A $\delta$ fiber reflex EMG failed to be induced. (B) The $C$-fiber reflex threshold in CClp $(n=10)$ rats was significantly decreased compared to that of normal rats (paired $t$-test, $* * P<0.0 I)$. (C) The $C$-fiber reflex latency in CClp rats was not different from that of normal rats (paired $t$-test). (D) The $C$-fiber reflex duration in CClp rats was not significantly different from that of normal rats (paired $t$-test). Stimulation: A $\delta$ or C-fiber threshold intensity stimulation.

Abbreviations: $\mathrm{CCl}$, chronic constriction injury of the sciatic nerve; CClp, chronic constriction injury of the partial sciatic nerve; EMG, electromyogram; Sti, stimulation.

preligation: $1.08 \pm 0.05 \mathrm{~mA}, P<0.01)$. As seen from Figure $5 \mathrm{C}$ and D, CCIp modeling did not significantly alter the EMG latency following Tc stimulation (CCIp: $177.40 \pm 13.22 \mathrm{~s}$ preligation: $152.79 \pm 6.68 \mathrm{~s}$ ) or the duration of EMG response (CCIp: $179.24 \pm 16.64$ s; preligation: $181.57 \pm 14.42 \mathrm{~s}$ ). The above data suggest that ligation of the sciatic nerve trunk in CCI not only impaired myelinated afferent fibers but also the corresponding motor nerve, leading to dystrophy of the innervated muscles, whereas the branch ligation in CCIp did not entirely impair the sciatic motor nerve and its innervated muscles.

\section{EA at GB30 increased pain threshold and latency in $\mathrm{CCl}$ and $\mathrm{CClp}$ rats}

We aimed to determine the effect of acupuncture on neuropathic mechanical pain in CCIp model. As shown in Figure 3A, in CCI $(n=7)$ rats, EA at GB30 improved the PMWL significantly (pre-EA: $7.02 \pm 0.60 \mathrm{~s}$; post-EA: $10.44 \pm 0.82 \mathrm{~s}, P<0.01)$; EA at GB30 also significantly increased the PMWL in CCIp $(n=10)$ rats (pre-EA: $7.75 \pm 0.55$ s; post-EA: $11.10 \pm 0.68 \mathrm{~s}, P<0.01)$. As can be seen from Figure 3B, in CCIp rats, EA at GB30 significantly increased PMWT (pre-EA: $15.70 \pm 1.12 \mathrm{~g}$; post-EA: $22.72 \pm 1.53 \mathrm{~g}$, $P<0.01$ ), similarly, a significant improvement was found in CCI rats (pre-EA: $14.35 \pm 1.20 \mathrm{~g}$; post-EA: $21.35 \pm 1.60 \mathrm{~g}$, $P<0.001)$. These data suggest that EA at GB30 is capable of alleviating neuropathic mechanical pain in either CCI or CCIp rats.

\section{EA at GB30 decreased the C-fiber reflex EMG in CClp rats}

To further test the effect of EA (conditioned stimulus) with different intensities at GB30 on nocuous stimulation-induced nociceptive responses, we applied EA of noxious or nonnoxious intensities as $0.8 \mathrm{Ta}, 1 \mathrm{Ta}, 1 \mathrm{Tc}$, and $2 \mathrm{Tc}$ to see the effect on $1.2 \mathrm{Tc}$-triggered $\mathrm{C}$-fiber reflex EMG in normal rats $(n=10)$ or CCIp $(n=10)$ rats. As shown in Figure 6A, $0.8 \mathrm{Ta}$ intensity of EA inhibited C-fiber reflex EMG at 0 and 1 minute in CCIp rats and at 0 minute in normal rats, but excited it at 4 minutes in normal rats $(P<0.05, P<0.01)$. Figure 6B 
A

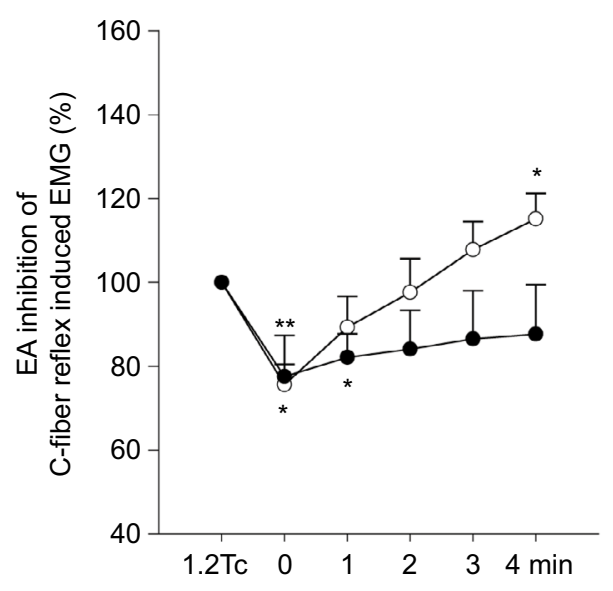

C

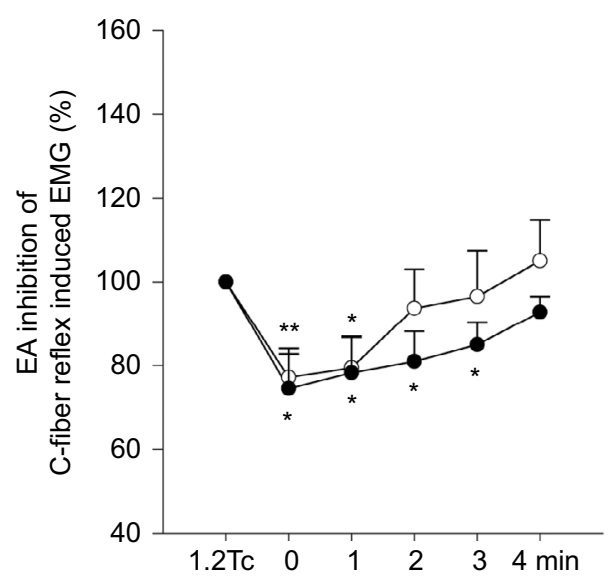

B

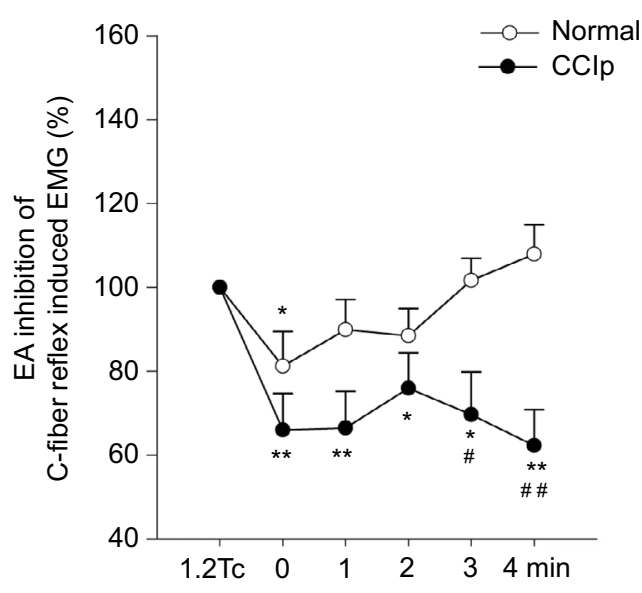

D

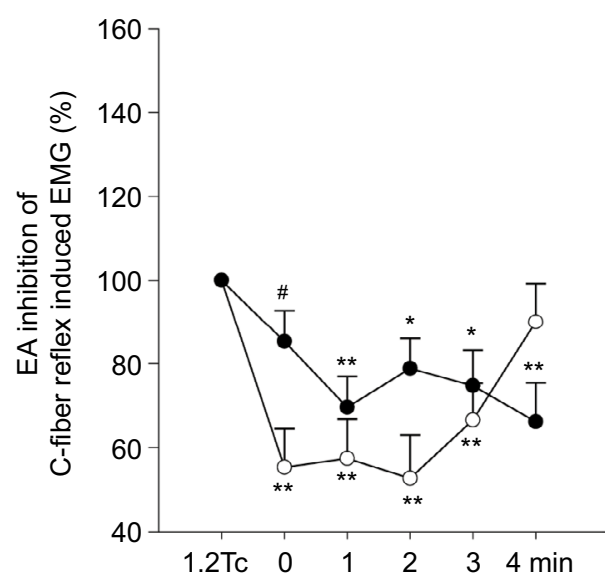

Figure 6 Effects of EA with different intensities at GB30 on EMG induced by I.2Tc (Tc, C-fiber threshold) stimulation intensity.

Notes: (A) EMG induced by the C-fiber reflex was significantly decreased at 0 and I minute after EA at GB30 with a stimulation intensity of 0.8 Ta in CClp ( $\mathrm{n}=10$ ) rats. EMG induced by the $\mathrm{C}$-fiber reflex was significantly decreased at 0 minute after EA at GB30 with a stimulation intensity of 0.8 Ta in normal rats, but excited it at 4 minutes in normal rats $(n=10)\left(* P<0.05\right.$, ${ }^{* * P}<0.01$, two-way ANOVA). (B) EMG induced by the C-fiber reflex was significantly decreased at $0-4$ minutes after EA at GB30 with a stimulation intensity of I Ta in CClp rats. EMG induced by the $\mathrm{C}$-fiber reflex was significantly decreased at 0 minute after $E A$ at GB30 with a stimulation intensity of I Ta in normal rats $\left({ }^{*} P<0.05,{ }^{* *} P<0.01\right.$, two-way ANOVA). At 3 and 4 minutes, EMG induced by the $C$-fiber reflex in $C C l p$ rats was lower than that in normal rats $\left({ }^{\#} P<0.05\right.$, ${ }^{\#} P<0.01$, two-way ANOVA). (C) EMG induced by the C-fiber reflex was significantly decreased at $0-3$ minutes after EA at GB30 with a stimulation intensity of I Tc in CClP rats. EMG induced by the $C$-fiber reflex was significantly decreased at 0 and I minute after $E A$ at GB30 with a stimulation intensity of I Tc in normal rats $(* P<0.05$, $* * P<0.0 I$, two-way ANOVA). (D) EMG induced by the C-fiber reflex was significantly decreased at I-4 minutes after EA at GB30 with a stimulation intensity of 2 Tc in CClp rats. EMG induced by the C-fiber reflex was significantly decreased at 0-3 minutes after EA at GB30 with a stimulation intensity of 2 Tc in normal rats ( ${ }^{*} P<0.05$, $* * P<0.01$, two-way $\left.A N O V A\right)$. Abbreviations: ANOVA, analysis of variance; CClp, chronic constriction injury of the partial sciatic nerve; EA, electroacupuncture; EMG, electromyogram; Ta, A $\delta$ fiber reflex threshold; Tc, C-fiber reflex threshold.

displays that 1 Ta EA significantly decreased $\mathrm{C}$ reflex EMG at $0,1,2,3$, and 4 minutes in CCIp rats but only at 0 minute in normal rats $(P<0.05, P<0.01)$. At 3 and 4 minutes, $\mathrm{C}$-fiber reflex EMG in CCIp rats was significantly lower than that in normal rats $(P<0.05, P<0.01)$. As shown in Figure $6 \mathrm{C}, 1$ Tc EA significantly reduced $\mathrm{C}$-fiber reflex EMG at $0,1,2$, and 3 minutes in CCIp rats but only at 0 and 1 minute in normal rats $(P<0.05, P<0.01)$. As can be seen in Figure 6D, the EMG response was significantly reduced by $2 \mathrm{Tc} E A$ at
GB30 at 1, 2, 3, and 4 minutes in CCIp rats but at $0,1,2$, and 3 minutes in normal rats $(P<0.05, P<0.01)$, and the EA inhibition at 0 minute was better in normal rats than that in CCIp rats $(P<0.05)$. These results suggest that EA at GB30 could alleviate nociceptive stimulation-induced EMG. The finding that EA of different intensities except 2 Tc at GB30 exhibited longer-lasting effects on 1.2 Tc-induced EMG in CCIp rats than those in normal rats implies a more significant acupuncture analgesia effect in a chronic pain model. 


\section{Discussion}

In the present study, we found that ligation of the common peroneal and tibial branches of the sciatic nerve in rats was capable of inducing phenotypes of neuropathic pain and significantly decreasing PMWL and PMWT, similar to ligation of the sciatic nerve trunk. EMG of biceps femoris induced by the C-fiber reflex could be recorded on the 5th day after ligation in CCIp but not CCI rats. Acupuncture at GB30 significantly increased the PMWL and PMWT in both $\mathrm{CCI}$ and CCIp rats. The EMG of biceps femoris triggered by the $\mathrm{C}$-fiber reflex could be inhibited for more than 2 minutes by acupuncture at GB30 in CCIp rats.

Cutting the sciatic nerve axons has been established as a neuroma model to mimic symptoms of phantom limb pain in humans. ${ }^{10}$ However, in this model, the hyperalgesia and sensory abnormalities cannot be experimentally tested. To investigate underlying mechanisms of neuropathic pain, the CCI model was established. ${ }^{7}$ Following this animal model, several alternative models were established according to different diseases mimicked. For example, the pSNL model was established by ligation of the sciatic nerve at the upper thigh level. In this model, $1 / 3-1 / 2$ of the sciatic nerve is trapped in the ligature. ${ }^{8,13}$ To imitate the protrusion of lumbar intervertebral disc, the SNL model was generated by ligation of L5 spinal nerve to study pain-related emotion or pain memory. ${ }^{9}$ To imitate the nerve radicular pain caused by stenosis of the spinal canal or the intervertebral foramen, a model utilizing chronic compression of the dorsal root ganglion was established. ${ }^{11,12}$ To date, CCI is still the most common research model used to study neuropathic pain in animals. CCI rats demonstrate spontaneous pain, thermal hyperalgesia, mechanical hyperalgesia, and cold hyperalgesia after nerve ligation for 1-15 days, and display peak pain expression at day 14-15. Although pain induced by nerve ligation could last for 15-30 days, hyperalgesia decreases over time in this model. Therefore, the CCI model is best utilized to mimic the symptoms and behaviors of chronic neuropathic pain in human peripheral nerve injuries (tumor suppression, heavy metal poisoning, hypoxia, or metabolic abnormalities). ${ }^{24} \mathrm{~A}$ previous study on CCI demonstrated that nerve edema could be found in the compression region 10 days after nerve ligation in rats. The edema and demyelination were exhibited in either the injury area or the distal part of ligated nerve. Generally, demyelination was found in the large myelinated axons (A $\alpha$ and $A \beta$ ), but not in the small myelinated axons (A $\delta)$, and unmyelinated axons were not affected. Two weeks after nerve injury, the myelinated fibers including $\mathrm{A} \delta$ were totally lost in the distal part of the injured nerve and the unmyelinated fibers related to the proximal part decreased by $34 \%-84 \% .^{7}$ Our pain behavior data showed that both CCI and CCIp modeling could mimic neuropathic pain. However, in CCI rats, the reflex induced by $\mathrm{A} \delta$ or C-fiber stimulation could not be recorded 5 days after surgery, except for spontaneous EMG of the biceps femoris muscle. In CCIp rats, however, the C-fiber reflex-induced EMG could be preserved 5 days after nerve ligation. The biceps femoris of rats are dominantly innervated by L4-5 spinal nerves. ${ }^{25,26} \mathrm{Ta}$ or Tc stimulation electrodes were performed on the third and fourth toe which are the receptive fields of the common peroneal nerve and the sural nerve. Although the damaged common peroneal nerve could impair the A $\delta$ fiber due to common peroneal nerve demyelination in CCIp rats, ${ }^{7}$ the sural nerve, which contains $A \delta$ fibers and the motor nerve fiber, was unaffected. We were able to record C-fiber reflex-induced EMG from this threshold stimulation on day 5 after nerve ligation in CCIp rats. On the contrary, in CCI rats, the ligation of the trunk of the sciatic nerve entirely damaged all three branches and thus led to the absence of C-fiber reflex-induced EMG.

Primary sensory neurons synthesize neuroregulator peptides such as SP and CGRP which play important roles in pain modulation. ${ }^{27,28} \mathrm{CGRP}$ and SP are mainly expressed in small neurons with unmyelinated axons (C fibers) which are dominated by polymordal nociceptors. CGRP is also expressed in a group of medium-sized cells with finely myelinated (A $\delta$ fibers) axons dominated by high-threshold mechanoreceptors. ${ }^{29}$ Our study shows that expression of CGRP and SP was increased in the CCIp group in a similar manner to the CCI group. Chronic demyelination and nerve injury caused impairment of the $\mathrm{A} \delta$ and $\mathrm{C}$ fibers in CCI and CCIp models and can increase the expression of these two peptides, consistent with previous reports. ${ }^{30,31}$ Our behavior and immunohistochemistry data suggest that the CCIp model may be as good as the CCI model for studying the effects and mechanisms of acupuncture analgesia on chronic neuropathic pain in experimental animals.

The C-fiber reflex is a validated biceps femoris EMG response to nociceptive stimulation at the sciatic nerve and has been commonly employed in pharmacological as well as acupuncture analgesia studies. ${ }^{32}$ As shown in nociceptive behavior and immunohistochemistry, CCIp was as good as CCI model, whereas C-fiber reflex EMG could not be induced in CCI model due to the damage of myelinated fibers of the sciatic nerve. CCIp model was preferential to CCI model because C-fiber reflex EMG can be induced in 
this model to further evaluate neuropathic pain and acupuncture analgesia effects in vivo in electrophysiological studies.

GB30, a key acupoint for sciatica treatment, ${ }^{33}$ was used in this study. It is believed that deep acupuncture at GB30 may repair the damaged axons and somas by increasing the release of nerve growth factor and fructooligosaccharide. ${ }^{34}$ C-fiber reflex-induced EMG could be used to evaluate the inhibition of acupuncture on nociceptive responses. The finding that EA at GB30 could increase pain threshold as well as inhibit nociceptive reflex EMG in CCIp rats provides solid evidence that the CCIp model could be used to study acupuncture analgesia on neuropathic pain as well as its underlying mechanisms. In the future, we will further use this model to study the effects of EA of different stimulation intensities on nociceptive pain and try to elucidate the underlying biological mechanisms.

\section{Conclusion}

CCIp rats exhibited the same pain behavior and immunohistochemistry changes as CCI rats. Acupuncture at GB30 could alleviate pain behavior in both CCI and CCIp models. But $\mathrm{C}$-fiber reflex EMG, which is an objective and direct criterion in evaluation of pain and acupuncture analgesia, could only be induced in CCIp model, indicating CCIp model is preferential to the CCI model for experimentally studying acupuncture analgesia on chronic neuropathic pain and its responsible mechanisms.

\section{Acknowledgments}

This work was supported by National Natural Science Foundation of China granted to Kun Liu (No. 81303054), Xin-yan Gao (No. 81473778), and Bing Zhu (No. 81130063), and grants from International Science \& Technology Cooperation Program of China (Grant No. 2014DFG32700) and National Basic Research Program of China (973 Program, No. 2014CB543103). We thank Professor Yi-kuan Xie for his constructive suggestions during our experiment and Kristin Schoepfer for assistance with word polishing and grammar corrections.

\section{Author contributions}

All authors contributed toward data analysis, drafting and critically revising the paper and agree to be accountable for all aspects of the work.

\section{Disclosure}

The authors report no conflicts of interest in this work.

\section{References}

1. Haanpää M, Attal N, Backonja M, et al. NeuPSIG guidelines on neuropathic pain assessment. Pain. 2011;152(1):14-27.

2. Jensen TS, Baron R, Haanpää M, et al. A new definition of neuropathic pain. Pain. 2011;152(10):2204-2205.

3. Konstaninou K, Dunn KM. Sciatica: review of epidemiological studies and prevalence estimate. Spine. 2008;33(22):2464-2472.

4. Wang LX, Wang ZJ. Animal and cellular models of chronic pain. $A d v$ Drug Deliv Rev. 2003;55(8):949-965.

5. Kim KJ, Yoon YW, Chung JM. Comparison of three rodent neuropathic pain models. Exp Brain Res. 1997;113(2):200-206.

6. Challa SR. Surgical animal models of neuropathic pain: Pros and Cons. Int J Neurosci. 2015;125(3):170-174.

7. Bennett GJ, Xie YK. A peripheral mononeuropathy in rat that produces disorders of pain sensation like those seen in man. Pain. 1988;33(1): 87-107.

8. Seltzer Z, Dubner R, Shir Y. A novel behavioral model of neuropathic pain disorders produced in rats by partial sciatic nerve injury. Pain. 1990;43(2):205-218.

9. Kim SH, Chung JM. An experimental model for peripheral neuropathy produced by segmental spinal nerve ligation in the rat. Pain. 1992;50(3):355-363.

10. Wall PD, Devor M, Inbal FR, et al. Autotomy following peripheral nerve lesions: experimental anaesthesia dolorosa. Pain. 1979;7(2): $103-111$.

11. Hu SJ, Xing JL. An experimental model for chronic compression of dorsal root ganglion produced by intervertebral foramen stenosis in the rat. Pain. 1998;77(1):15-23.

12. Song XJ, Hu SJ, Greenquist KW, Zhang JM, LaMotte RH. Mechanical and thermal hyperalgesia and ectopic neuronal discharge after chronic compression of dorsal root ganglia. J Neurophysiol. 1999;82(6): $3347-3358$.

13. Decosterd I, Woolf CJ. Spared nerve injury: an animal model of persistent peripheral neuropathic pain. Pain. 2000;87(2):149-158.

14. Zhao XY, Zhang QS, Yang J, et al. The role of arginine vasopressin in electroacupuncture treatment of primary sciatica in human. Neuropeptides. 2015;52:61-65.

15. Li J, Dong JC, Yue JJ. Effects of acupuncture on default mode network images of chronic sciatica patients in the resting network state. Zhongguo Zhong Xi Yi Jie He Za Zhi. 2012;32(12):1624-1627.

16. Austin PJ, Wu A, Moalem-Taylor G. Chronic constriction of the sciatic nerve and pain hypersensitivity testing in rats. JVis Exp. 2012;(61):3393.

17. Falinower S, Willer JC, Junien JL, Le Bars D. A C-fiber reflex modulated by heterotopic noxious somatic stimuli in the rat. $J$ Neurophysiol. 1994;72(1):194-213.

18. Zhu B, Xu WD, Rong PJ, Ben H, Gao XY. A C-fiber reflex inhibition induced by electroacupuncture with different intensities applied at homotopic and heterotopic acupoints in rats selectively destructive effects on myelinated and unmyelinated afferent fibers. Brain Res. 2004;1011(2):228-237.

19. Taliyan R, Sharma PL. Possible mechanism of protective effect of thalidomide in STZ-induced-neuropathic pain behavior in rats. Inflammopharmacology. 2012;20(2):89-97.

20. Chen X, Yang BX, Liu H, Yang Y, Xiao H. Effects of intrathecal lornoxicam on withdrawal thresholds of rats to mechanical and thermal stimuli. Sichuan Da Xue Xue Bao Yi Xue Ban. 2007;38(4):667-670.

21. Deng CL, Yin KJ. Experimental Acupuncture Science. Beijing: People's Medical Publishing House; 1998:147-148.

22. Zhang RX, Li A, Liu B, et al. Electroacupuncture attenuates bonecancer-induced hyperalgesia and inhibits spinal preprodynorphin expression in a rat model. Eur J Pain. 2008;12(7):870-878.

23. Li J, Dong JC, Yue JJ. Effects of acupuncture on default mode network images of chronic sciatica patients in the resting network state. Zhongguo Zhong Xi Yi Jie He Za Zhi. 2012;32(12):1624-1627.

24. Guneli E, Onal A, Ates M. Effects of repeated administered ghrelin on chronic constriction injury of the sciatic nerve in rats. Neurosci Lett. 2010;479(3):226-230. 
25. Molander C, Grant G. Spinal cord projections from hindlimb muscle nerves in the rat studied by transganglionic transport of horseradish peroxidase, wheat germ agglutinin conjugated horseradish peroxidase, or horseradish peroxidase with dimethylsulfoxide. J Comp Neurol. 1987;260(2):246-255.

26. Hinterdorfer P, Parsaei B, Stieglbauer K. Segmental innervation in lumbosacral transitional vertebrae (LSTV): a comparative clinical and intraoperative EMG study. J Neurol Neurosurg Psychiatry. 2010;81(7):734-741.

27. Konishi S, Otsuka M. The effects of substance $P$ and other peptides on spinal neurons of the frog. Brain Res. 1974;65(3):397-410.

28. Morton CR, Hutchison WD. Release of sensory neuropeptides in the spinal cord: Studies with calcitonin gene-related peptide and galanin. Neuroscience. 1989;31(3):807-815.

29. McGaraughty S, Chu KL, Brown BS, et al. Contributions of central and peripheral TRPV1 receptors to mechanically evoked and spontaneous firing of spinal neurons in inflamed rats. $J$ Neurophysiol. 2008;100(6):3158-3166.
30. Caviedes-Bucheli J, Moreno JO, Ardila-Pinto J, et al. The effect of orthodontic forces on calcitonin gene-related peptide expression in human dental pulp. J Endod. 2011;37(7):934-937.

31. Ramer MS, Ma W, Murphy PG, Richardson PM, Bisby MA. Galanin expression in neuropathic pain: friend or foe? Ann N Y Acad Sci. 1998;863:390-401.

32. Guirimand F, Strimbu-Gozariu M, Willer JC, et al. Effects of mu, delta and kappa opioid antagonists on the depression of a C-fiber reflex by intrathecal morphine and DAGO in the rat. J Pharmacol Exp Therap.1994;269(3):1007-1020.

33. Zhao Y, Wang GL. Randomized controlled study on proximal needling for sciatica. Zhongguo Zhen Jiu. 2011;31(5):425-428.

34. Liu YL, Li Y, Ren L, et al. Effect of deep electroacupuncture stimulation of "Huantiao" (GB 30) on changes of function and nerve growth factor expression of the injured sciatic nerve in rats. Zhen Ci Yan Jiu. 2014;39(2):93-99.
Journal of Pain Research

\section{Publish your work in this journal}

The Journal of Pain Research is an international, peer reviewed, open access, online journal that welcomes laboratory and clinical findings in the fields of pain research and the prevention and management of pain. Original research, reviews, symposium reports, hypothesis formation and commentaries are all considered for publication.

\section{Dovepress}

The manuscript management system is completely online and includes a very quick and fair peer-review system, which is all easy to use. Visit http://www.dovepress.com/testimonials.php to read real quotes from published authors. 